\title{
Not all lesions are created equal: removal of a cavernous hemangioma
}

A 63-year-old woman was referred for evaluation of a subepithelial esophageal mass and dysphagia. Endoscopy at an outside hospital reported an increase in the size of the mass. Endoscopic ultrasound showed a hypoechoic well-defined subepithelial mass originating within the submucosa and measuring $23 \mathrm{~mm}$ in maximum diameter. Fine-needle aspiration of the mass was nondiagnostic. Owing to the dysphagia and the increase in size, we proceeded with endoscopic resection ( $\vee$ Video $\mathbf{1}$ ).

The patient was placed in the supine position and the procedure was performed under general anesthesia. A GIF-HQ190 endoscope (Olympus Corp., Center Valley, Pennsylvania, USA) fitted with a transparent cap was advanced and identified a subepithelial mass in the mid esophagus, extending $20 \mathrm{~cm}$ to $24 \mathrm{~cm}$ from the incisors ( $\mathbf{F i g} \mathbf{1}$ a). A submucosal injection of $0.9 \%$ saline and methylene blue was made approximately $2 \mathrm{~cm}$ proximal to the lesion. A hybrid multipurpose T-knife (Erbe, Marietta, Georgia, USA) was used to make a $1.5-\mathrm{cm}$ mucosal incision to create an entry point to the submucosal space. The submucosal layer was visualized with blue hue and further injected. The lateral margins were then carefully dissected with an insulated-tip knife IT2 (Olympus Corp.), in a proximal to distal direction. Using the pocket technique, dissection of the submucosal space was performed to expand the tunnel down to the distal end of the esophageal mass (settings: endocut $Q$ current effect 3 , interval 1 , period 1 , soft coag

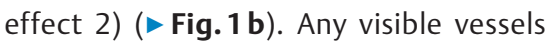
or bleeding were treated by coagulation using the hybrid knife or coagulation graspers. The lesion was then completely removed en bloc ( $\triangleright$ Fig. $\mathbf{1} \mathbf{c}, \mathbf{d}$ ). The defect was closed using a 2.0 polypropylene suture in a running suture pattern from the distal to the proximal end of

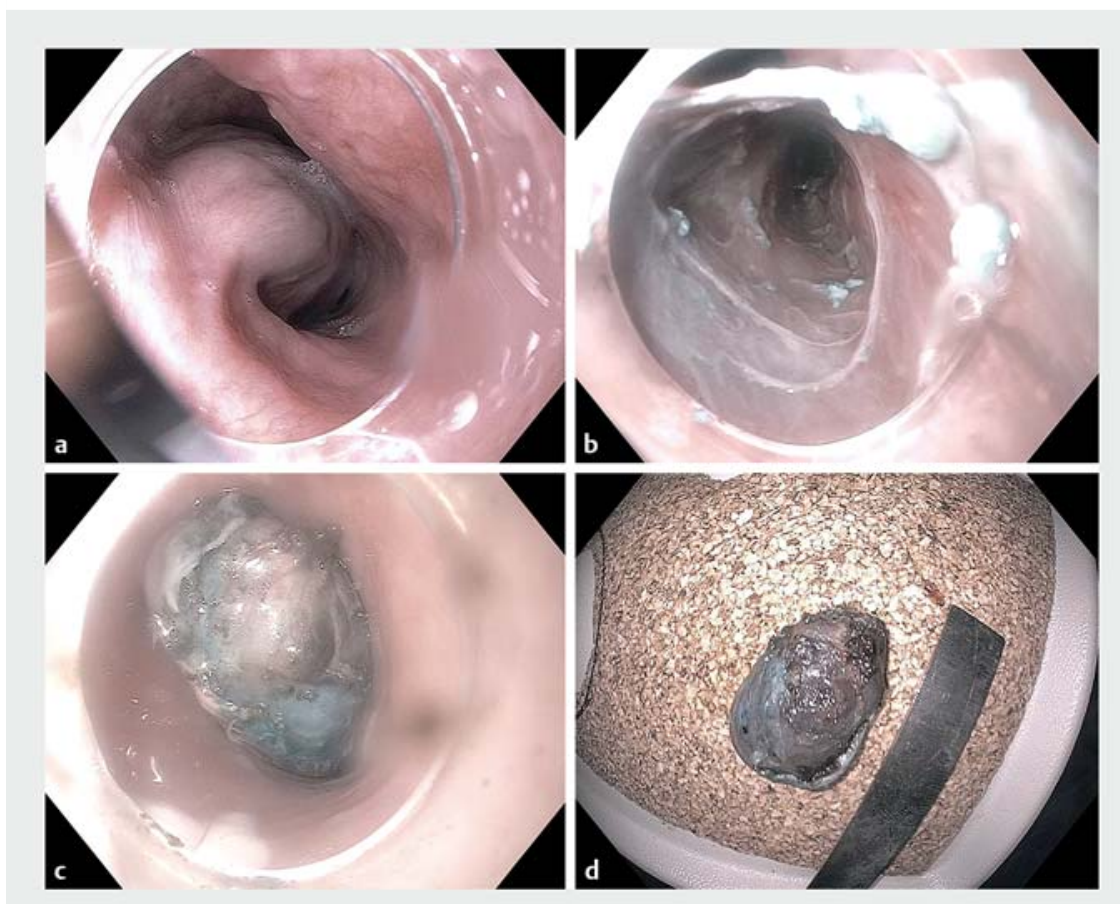

- Fig. 1 Endoscopic images. a The esophageal subepithelial lesion. b The submucosal tunnel after dissection. $\mathbf{c}$ The subepithelial lesion after complete en bloc resection. $\mathbf{d}$ The subepithelial lesion after retrieval, measuring $4 \mathrm{~cm}$.

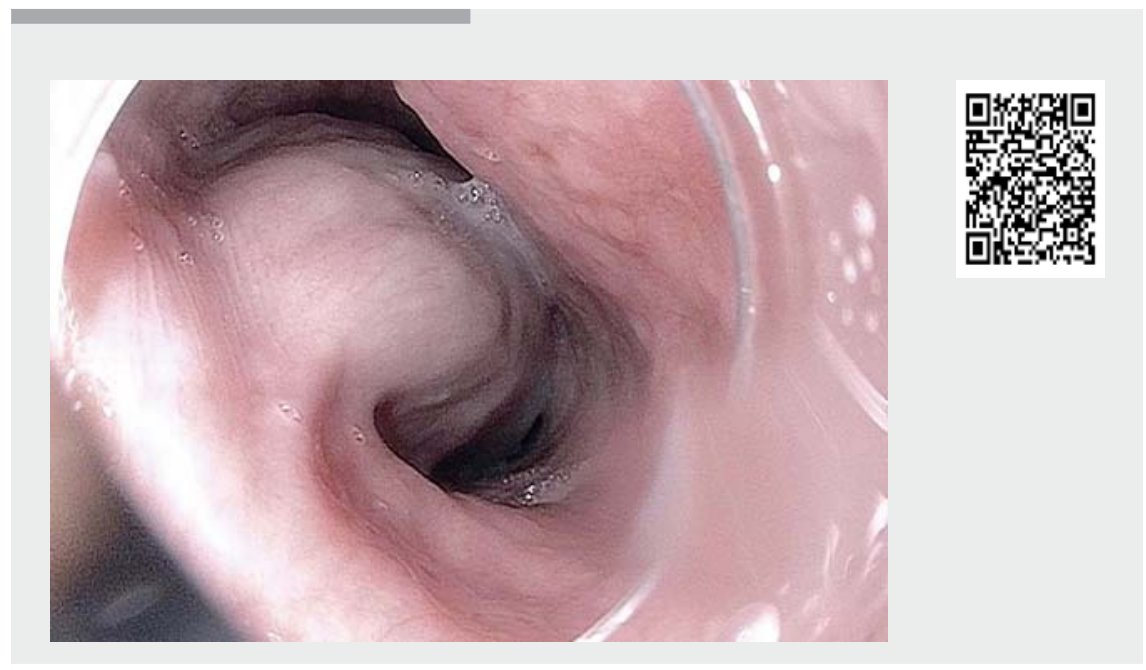

$\checkmark$ Video 1 Endoscopic submucosal dissection with complete en bloc removal of an esophageal cavernous hemangioma. 


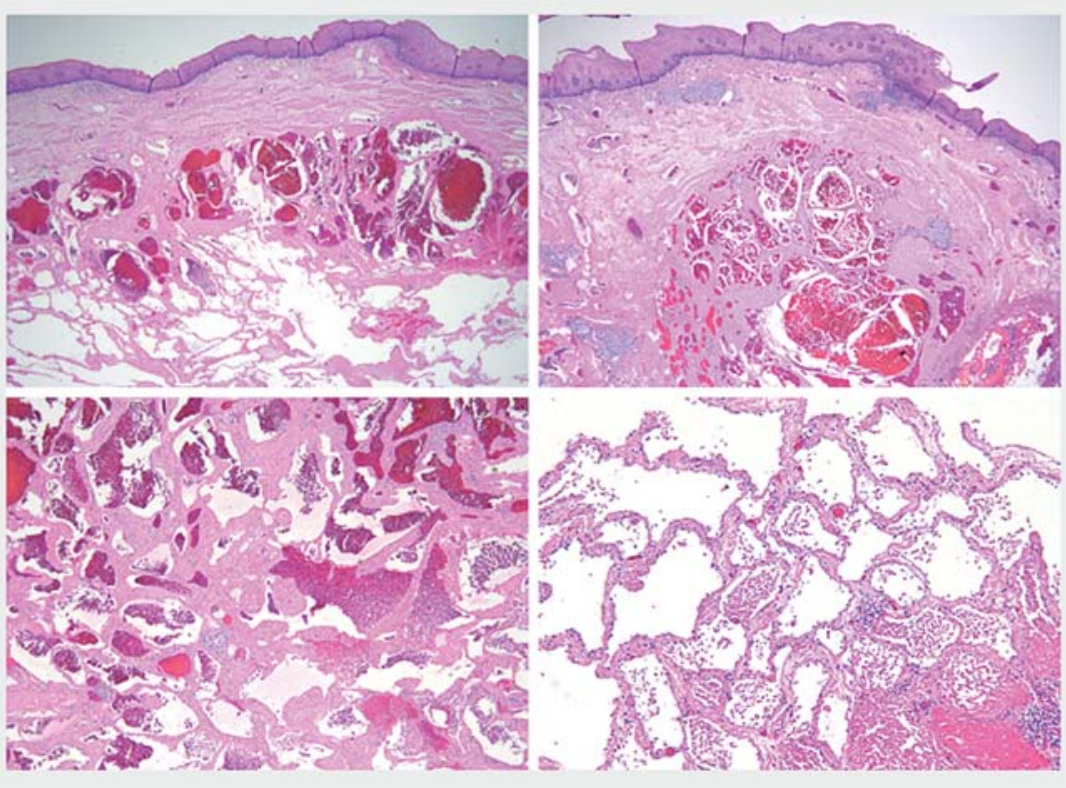

- Fig. 2 The low-power examination (top row) showed benign squamous mucosa with a submucosal-based benign vascular proliferation. Higher-power examination (lower row) showed large cystically dilated vessels with thin, focally fibrotic walls and a benign, flattened, endothelial lining.

the lesion. A cinching device was then used to cinch the suture. Finally, Duraclips (ConMed Corp., Utica New York, USA) were used to reinforce closure of the proximal defect.

Low-power microscopic examination showed benign squamous mucosa with a submucosal-based benign vascular proliferation. Higher-power examination revealed large cystically dilated vessels with thin, focally fibrotic walls and a benign, flattened, endothelial lining, which was consistent with cavernous hemangioma (> Fig. 2).

Barium swallow study showed no leak and the patient tolerated a regular diet. She was discharged home in a good condition and was completely asymptomatic at 1 month follow-up.

Cavernous hemangioma in the esophagus is a rare benign tumor. Small lesions are usually asymptomatic, but large tumors can often cause dysphagia. Histopathological examination establishes a definitive diagnosis, as radiographic and

ciplinary therapeutic approach $[1,2]$. symptoms.

Endoscopy_UCTN_Code_TTT_1AO_2AG

\section{Competing interests} Medtronic.

The authors

Qais Dawod, Danny Issa, Reem Z. Sharaiha Division of Gastroenterology and Hepatology, New York Presbyterian Hospital/Weill Cornell Medical Center, New York, New York, United States
Corresponding author

Reem Z. Sharaiha, MD

Division of Gastroenterology and Hepatology, Department of Medicine, Weill Cornell Medicine, 1305 York Avenue, 4th Floor, New York, NY 10021, United States Fax: +1-646-962-0110 rzs9001@med.cornell.edu

\section{References}

[1] Imenpour H, Muti M, Pastorino G. Oesophageal cavernous haemangioma. Pathologica 2018; 110: 72-74

[2] Santamaria-Barria JA, Banki F, Rajendran S et al. Hemangioma of the cervical esophagus: a rare case of dysphagia mimicking a large esophageal polyp on endoscopy. J Thorac Cardiovasc Surg 2016; 152: e59-61

Bibliography

DOI https://doi.org/10.1055/a-1024-3410

Published online: 2.12.2019

Endoscopy 2020; 52: E154-E155

(c) Georg Thieme Verlag KG

Stuttgart · New York

ISSN 0013-726X

Complete en bloc removal can be successfully achieved using endoscopic submucosal dissection, with resolution of

Dr. Sharaiha is a consultant for Boston Scientific, Olympus, Apollo, Cook Endoscopy and
This section has its own submission website at

https://mc.manuscriptcentral.com/e-videos 